\title{
ON PARTIAL SUMS OF LAGRANGE'S SERIES WITH APPLICATION TO THE THEORY OF QUEUES
}

\author{
P. D. FINCH \\ (received 6 December 1962)
}

\section{Introduction}

Lagrange's theorem on the reversion of power series may be stated in the following form (e.g. Whittaker and Watson [3]).

THEOREM L. Let $k(z)$ be a function of $z$ analytic on and inside a contour $C$ surrounding $a$ point $x$ and let $y$ be such that for all points $z$ on $C$

$$
|y k(z)|<|z-x| .
$$

Then the equation

$$
z=x+y k(z),
$$

regarded as an equation in $z$, has one root, $\zeta$, in the interior of $C$ and if $\phi(z)$ is any function of $z$ analytic on and inside $C$

$$
\phi(\zeta)=\phi(x)+\sum_{m=1}^{\infty} \frac{y^{m}}{m !} \frac{d^{m-1}}{d x^{m-1}}\left[\{k(x)\}^{m} \phi^{\prime}(x)\right] .
$$

By a partial sum of Lagrange's series we mean an expression of the form

$$
\phi^{n}(x)=\phi(x)+\sum_{m=1}^{n} \frac{y^{m}}{m !} D^{m-1}\left[\{k(x)\}^{m} D \phi(x)\right]
$$

where $D \equiv d / d x$. In this paper we consider expressions $\phi_{j}^{n}(x)$ of the form (1.4) where $\phi(x)=\phi_{j}(x)=x^{j}, x \geqq 0, j \geqq 0$. We show that the $\phi_{j}^{n}(x)$ satisfy a certain set of difference equations which occur in the theory of queues. This result gives a simple proof of a conjecture of Finch [2] which has since been proved by Brockwell [l] using quite different methods.

\section{The difference equation of partial sums}

We prove the following

THEOREM. Let

$$
k(z)=\sum_{j=0}^{\infty} k_{j} z^{j}
$$


be analytic in some region surrounding the point $x$, and let

$$
\phi_{j}^{n}(x)=\phi_{j}(x)+\sum_{m=1}^{n}\left(y^{m} / m !\right) D^{m-1}\left[\{k(x)\}^{m} D \phi_{j}(x)\right]
$$

where $\phi_{j}(x)=x^{j}$ and $D \equiv d / d x$.

Then, $\phi_{0}^{n}(x) \equiv 1$ and

$$
\phi_{j}^{n+1}(x)-x \phi_{j-1}^{n+1}(x)=y \sum_{i=0}^{\infty} k_{i} \phi_{j+i-1}^{n}(x), \quad j \geqq 1, n \geqq 1 .
$$

Proof. By definition $\phi_{0}^{n}(x)=\phi_{0}(x) \equiv 1$. For $j \geqq 1$ we obtain from (2.2)

$$
y \sum_{i=0}^{\infty} k_{i} \phi_{j+i-1}^{n}(x)=x \phi_{j-1}(x)-x \phi_{j-1}^{n+1}+\sum_{m=1}^{\infty} \frac{y^{m}}{m !} \psi_{j}^{m}(x),
$$

where

$$
\psi_{j}^{m}(x)=x D^{m-1}\left[\{k(x)\}^{m} D \phi_{j-1}(x)\right]+m D^{m-2}\left[\{k(x)\}^{m-1} D\left\{\phi_{j-1}(x) k(x)\right\}\right] .
$$

Expanding the terms on the right of (2.5) by Leibnitz' theorem and rearranging we obtain

$$
\psi_{j}^{m}(x)=\sum_{s=0}^{m-1}\left(\begin{array}{c}
m-1 \\
s
\end{array}\right)\left[D^{s}\{k(x)\}^{m}\right]\left[x D^{m-3} \phi_{j-1}(x)+(n-s) D^{n-1-s} \phi_{j-1}(x)\right] .
$$

It can be verified readily or proved easily by induction that

$$
x D^{m-s} \phi_{j-1}(x)+(m-s) D^{m-1-s} \phi_{j-1}(x)=D^{m-1} \phi_{j}(x) .
$$

Thus from $(2.6)$

$$
\psi_{j}^{m}(x)=D^{m-1}\left[\{k(x)\}^{m} D \phi_{j}(x)\right]
$$

Substituting in (2.4) we obtain (2.3). This proves the theorem.

COROLlaRY. If $k(z)$ is analytic at $z=0$ then the quantities

$$
\phi_{j}^{n}=(j / m) \sum_{m=j}^{n}\{(n-j) !\}^{-1}\left[D^{m-j}\{k(x)\}^{m}\right]_{x=0}, \quad n \geqq j \geqq 1,
$$

satisfy the equations

$$
\phi_{j}^{n+1}=\sum_{i=0}^{\infty} k_{i} \phi_{j+i-1}^{n}
$$

with $\phi_{0}^{n} \equiv 1$ and $\phi_{j}^{n}=0,1 \leqq n<j$ and initial conditions $\phi_{0}^{1}=1, \phi_{1}^{1}=k_{0}$, $\phi_{j}^{1}=0, j>1$.

The corollary is proved easily by putting $x=0, y=1$ in the theorem. 


\section{Application to the queueing system $G I / M / 1$.}

Consider the queueing system $G I / M / 1$ in which the times at which customers arrive form a renewal process with inter-arrival distribution $A(x)$ and in which the service-time distribution is exponential with parameter $\mu$. Let $P_{\xi}^{n}$ be the probability that the $n$th arrival finds $j$ customers in the system, then

$$
P_{j}^{n+1}=\sum_{i=0}^{\infty} k_{i} P_{j+i-1}^{n}, \quad n \geqq 1, j \geqq 1
$$

where

$$
k_{m}=(m !)^{-1} \int_{0}^{\infty}(\mu x)^{m} e^{-\mu x} d A(x)
$$

and

$$
k(z)=\sum_{m=0}^{\infty} k_{m} z^{m} \text { is analytic at } z=0 .
$$

Write $Q_{j}^{n}=\sum_{m=j}^{\infty} P_{m}^{n+1}$, then $Q_{j}^{n}$ is the probability that the $(n+1)$ the arrival finds $j$ or more customers in the system, and from (3.1)

$$
Q_{j}^{n+1}=\sum_{i=0}^{\infty} k_{i} Q_{j+i-1}^{n}, \quad n \geqq 1, j \geqq 1,
$$

with $Q_{0}^{n} \equiv 1$.

If the queueing system starts from emptiness, so that $P_{0}^{1}=1, P_{j}^{1}=0$, $j>0$, we have $Q_{0}^{1}=1, Q_{1}^{1}=k_{0}$ and $Q_{j}^{1}=0 j>1$. Since the $Q_{j}^{n+1}$ are uniquely determined by the $Q_{j}^{n}$ we can appeal to the corollary of the theorem of the last section and deduce

$$
Q_{j}^{n}=(j / m) \sum_{m=j}^{n}\{(m-j) !\}^{-1}\left[D^{m-j}\{k(x)\}^{m}\right]_{x=0}, \quad n \geqq j \geqq 1 .
$$

When $k^{\prime}(1)>1$ a limiting distribution of queue size exists and $Q_{j}^{n} \rightarrow q^{j}$, $j \geqq 1$ where $0<q<1$ and $q$ is the only root within the unit circle of the equation $z=k(z)$. When $k^{\prime}(1) \geqq 1$ there is no root of this equation within the unit circle and a limiting distribution of queue size does no exist, in this case $Q_{j}^{n} \rightarrow 1, j \geqq 1$.

Equation (3.3) establishes the conjecture of Finch [2] that the probabilities $Q_{j}^{n}$ are given by the partial sums of the Lagrange series for $q^{j}$. As noted earlier this result has been proved by Brockwell, [1].

\section{References}

[1] Brockwell, P. J., The transient behaviour of the queueing system GI/M/1, Journ. Aust. Math. Soc.

[2] Finch, P. D., The single Server queueing system with non-recurrent input process and Erlang Service time, Journ. Aust. Math. Soc.

[3] Whittaker, E. T. and Watson, G. N., Modern Analysis (Cambridge Univ. Press, 1950).

University of Melbourne. 